\title{
Specific features of the practical implementation of observation planning in systems for monitoring networks with dynamic topology
}

\author{
Pavel Razumov ${ }^{1, *}$, Nikolay Boldyrikhin ${ }^{1}$, Larissa Cherckesova $^{1}$, Olga Safaryan ${ }^{1}$, Irina \\ Reshetnikova $^{1}$, and Andrey Beryoza ${ }^{2}$ \\ ${ }^{1}$ Don state technical University Rostov-on-Don, 1, Gagarin square, Russia \\ ${ }^{2}$ Don state technical University Shakhty, 147, Shevchenko str., Rostov region, Russia
}

\begin{abstract}
The article is devoted to the issues of practical implementation of planning observations of information processes flows, the sources of which are dynamic network objects that can appear in the network at random times. A variant of the implementation of the suboptimal distribution of time resources of the monitoring system between the tasks of detecting and evaluating random processes that appear in accordance with the laws of the Poisson flow is proposed.
\end{abstract}

\section{Introduction}

In many branches of modern technology, information surveillance systems are of great importance [1-7]. Typical tasks that such systems solve are the tasks of monitoring communication networks [4], ensuring the security of information in computer systems and networks [6], monitoring the trajectories of air and ground transport [2,3,5,7], etc. Optimization of the operating modes of such systems can significantly increase the efficiency of their use.

Of practical interest are the issues of observation management, taking into account the probabilistic nature of the appearance of information processes [1-7], since this allows you to remove a number of severe limitations inherent in classical algorithms for planning an experiment. It is these operating conditions that correspond to monitoring systems for networks with a dynamic topology, in which network objects can appear at random times. Such objects are sources of information processes that can be described by dynamic models [4], and, therefore, the classical mathematical apparatus for controlling observations can be applied to such problems [8-11].

Of great importance is the model that describes the regularity of the appearance of the evaluated processes at the input of the information system. Within the framework of the article, it is assumed that information processes appear in accordance with the laws of the Poisson flow. In [1] the mathematical formulation of such a problem and the procedure for its solution are presented in detail. At the same time, it seems relevant to study the features

\footnotetext{
* Corresponding author: therazumov@gmail.com
} 
of the practical application of observation plans obtained as a result of the implementation of the procedure given in [1].

Suppose that as a result of the implementation of the iterative procedure [1], an observation plan is synthesized (Fig. 1 a). This plan is a priori, and involves the observation of two processes in accordance with the given probabilistic characteristics of the moments of occurrence of such processes at the input of the information system. The probability of the appearance of the third process during the observation interval is so small that the program function associated with it does not affect the formation of the observation plan [1]. However, in real observation mode, the third and other information processes may appear, i.e. the obtained a priori plan can be used only as a reference plan, which must be corrected directly during observations.

It should be noted that the approach proposed in [1] can be used in information systems with time division of resources, ie. at the same moment in time, either one of the information processes can be evaluated, or new processes can be detected. The allocation of time resources in such an information system occurs by allocating quanta of machine time, and the transition from one task to another can be carried out only after the completion of the next quantum (Fig. 1 b).

It is assumed that the minimum frequencies of using quanta, spent on detection $f_{\text {min }} s$ and on estimation of one information process $f_{\text {min } o}$, are determined, at which a satisfactory solution of the problems of detection and estimation is provided. The surplus resource is determined by the expression

$$
f_{\text {ex }}=f_{\text {max }}-f_{\text {min } s}-f_{\text {mino }} * \Lambda,
$$

where $f_{e x}$ is the repetition rate of quanta available for suboptimal distribution between tasks; $f_{\text {max }}$ - the maximum repetition rate of quanta, determined by the characteristics of the information system;

$$
\Lambda=\left\{\begin{array}{c}
0, \text { if no process detected; } \\
I-1, \text { if at least one process detected, } I=1,2,3 \ldots, I^{\prime}
\end{array}\right.
$$

$I$ - the number of detected processes at the current time (the meaning of parameter will be explained below).

Let us consider in more detail the procedure for adjusting the a priori plan directly during the observation process. 


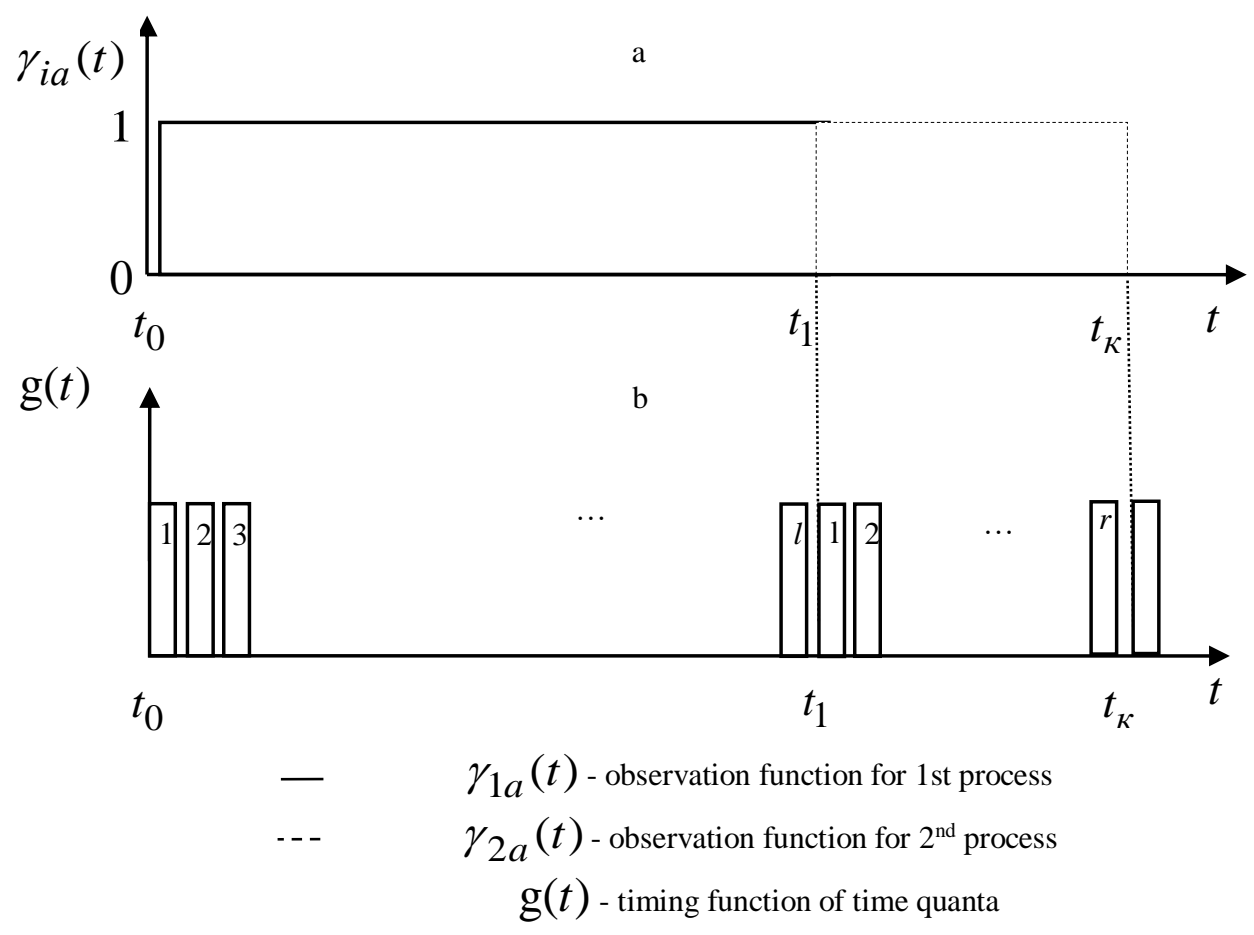

Fig. 1. Apriori observation plan.

Suppose that at some point in time $t^{1} \in\left[t_{0}, t_{1}\right)$ the detection of the 1 st information process occurred, then starting from this moment in time the resource of the information system is distributed between the tasks of evaluating the 1st process and detecting the following processes. In this case, the number of quanta used for detection is determined by the frequency $f_{\min } s$, the remaining quanta are spent on estimation. Let us call this mode "maximum".

If at some point in time $t^{2} \in\left[t_{0}, t_{1}\right)$ the detection of the 2nd process occurred, then starting from this moment in time the resource of the information system is distributed between the tasks of evaluating the 1 st and 2 nd information processes and detecting the following processes. In this case, the number of quanta used for detection is determined by frequency $f_{\min s}$, the number of quanta used for evaluating the 2 nd process is determined by $f_{\text {min o }}$ ("minimum" estimation mode), the rest are spent on evaluating the first process. This is due to the fact that according to the a priori plan (Fig. $1 \mathrm{a}$ ), the interval $\left[t_{0}, t_{1}\right)$ is defined as the interval of priority assessment of the first information process. Let's call this mode "priority".

If one or more information processes are detected on interval $\left[t_{0}, t_{1}\right)$, then all of them will be evaluated in the "minimum" mode, i.e. the frequency of the allocated quanta will be determined by the value $f_{\text {min } o}$.

Thus, if the interval $\left[t_{0}, t_{1}\right)$ contains only 


$$
l=f_{\max } *\left(t_{1}-t_{0}\right)
$$

quanta, then of them for detection will be spent

$$
s=f_{\min s} *\left(t_{1}-t_{0}\right)
$$

quanta, the estimation of all processes, except the first, will be spent

$$
o=f_{\min o} *\left(t_{1}-t_{0}\right) *(I-1)
$$

quanta. The evaluation of the first process in priority mode will use all the remaining

$$
e=l-s-o=f_{e x} *\left(t_{1}-t_{0}\right)
$$

quanta.

Obviously, when the value $I$ exceeds a certain threshold value

$$
I^{\prime}=\left\lfloor\frac{\left(f_{\max }-f_{\min s}-f_{\min o}\right)}{f_{\min o}}\right\rfloor+1
$$

a situation arises when $f_{\text {ex }} \leq f_{\text {min } o}$. In this case, the detection is suspended and the entire resource of the information system is distributed among the detected information processes.

On time interval $\left[t_{1}, t_{\kappa}\right]$, according to the a priori plan, the second process is accompanied in the "priority" mode, the rest in the "minimum" mode. The distribution of resources in this case is similar to that considered above, and expressions (2) - (5), respectively, take the form

$$
\begin{gathered}
r=f_{\text {max }} *\left(t_{\kappa}-t_{1}\right), \\
s=f_{\text {min } s} *\left(t_{\kappa}-t_{1}\right) \\
o=f_{\text {min } o} *\left(t_{\kappa}-t_{1}\right) * I \\
e=r-s-o=f_{\text {ex }} *\left(t_{\kappa}-t_{1}\right),
\end{gathered}
$$

where $r$ is the number of quanta that fit in the interval $\left[t_{1}, t_{\kappa}\right]$. 


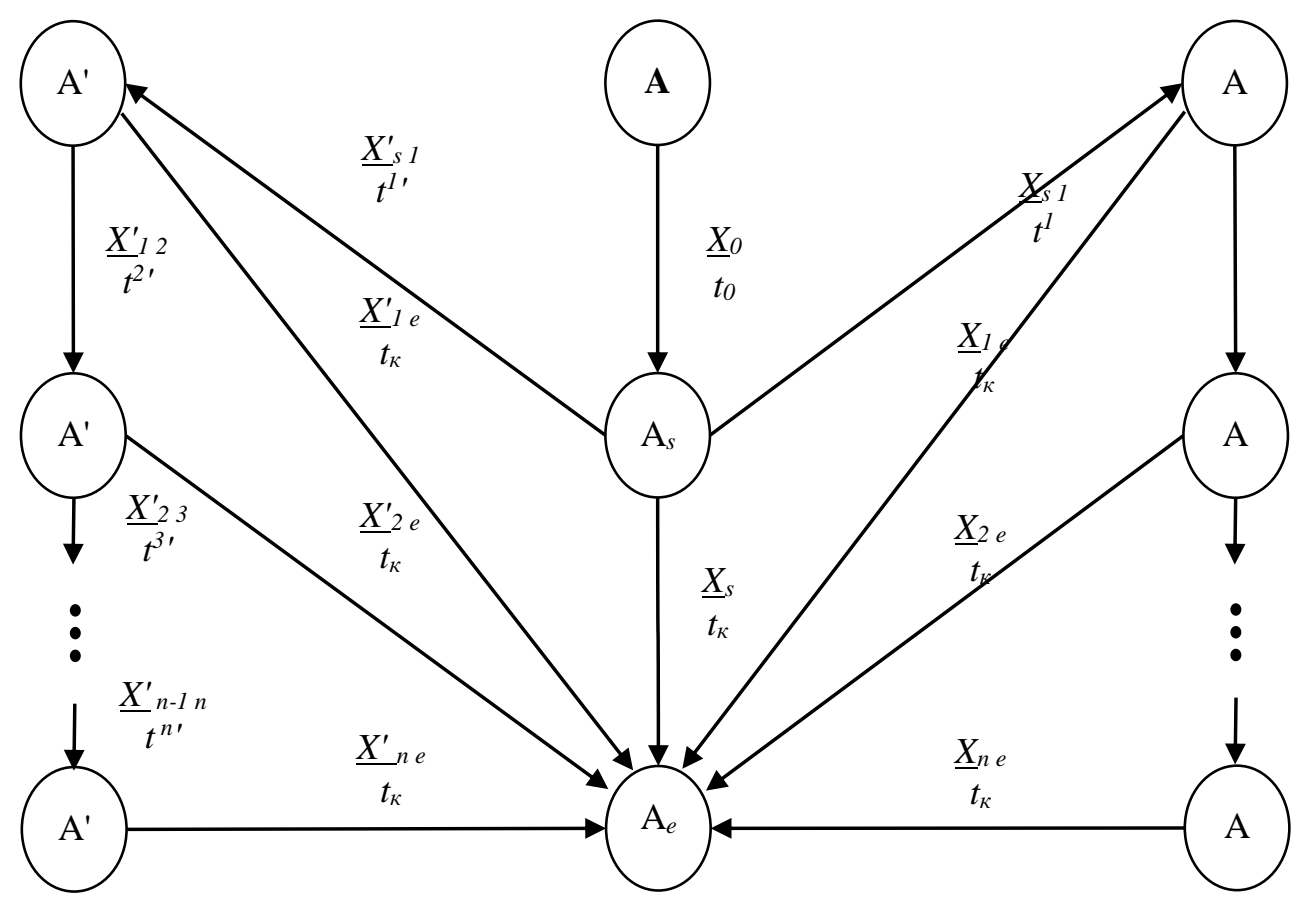

Fig. 2. Graph of abstract Mealy automaton.

For the practical implementation of correcting the observation plan, we use the theory of discrete automata [8]. Figure 2 shows the graph of an abstract Mealy automaton, which implements the adaptation of the a priori plan (Figure 1 a) to real conditions directly during observations.

Figure 2 introduces the following notations:

$A_{O}$ - the initial state of the machine, corresponding to the inclusion of the information system;

$A_{S}$ - the state of the machine corresponding to the mode of "pure" detection of information processes, i.e. all quanta are spent on detection;

$A_{1}$ - the state of the automaton corresponding to the estimation mode of the 1 st information process in the "maximum" mode;

$A_{2}$ - the state of the machine corresponding to the evaluation of the 1 st information process in the "priority" mode, and the 2nd process - in the "minimum";

$A_{n}$ - the state of the automaton corresponding to the evaluation of the 1 st information process in the "priority" mode, and the other processes (2nd, 3rd, ... nth) - in the "minimum";

$A_{1}^{\prime}$ - the state of the automaton corresponding to the estimation mode of the 1 st informational one in the "minimum" mode, the remaining quanta are spent on detection;

$A_{2}^{\prime}$ - the state of the machine, corresponding to the evaluation mode of the 2 nd informational mode in the "priority" mode, and the 1 st - in the "minimum" mode; 
$A_{n}^{\prime}$ - the state of the automaton corresponding to the evaluation of the 2 nd information process in the "priority" mode, and the rest of the processes (1st, 3rd, ..., nth) in the "minimum";

$A_{e}$ - the state of the machine, corresponding to the shutdown of the information system;

$X_{O}$ - input signal to enable the mode $A_{S}$;

$X_{s 1}\left(X_{s 1}^{\prime}\right)$ - input signal for switching from state $A_{s}$ to state $A_{1}\left(A_{1}^{\prime}\right)$;

$X_{12}\left(X_{12}^{\prime}\right)$ - input signal for switching from state $A_{1}\left(A_{1}^{\prime}\right)$ to state $A_{2}\left(A_{2}^{\prime}\right)$;

$X_{23}\left(X_{23}^{\prime}\right)$ - input signal for switching from state $A_{2}\left(A^{\prime}{ }_{2}\right)$ to state $A_{3}\left(A_{3}^{\prime}\right)$;

$X_{n-1 n}\left(X_{n-1 n}^{\prime}\right)$ - input signal for switching from state $A_{n-1}\left(A_{n-1}^{\prime}\right)$ to state $A_{n}\left(A_{n}^{\prime}\right)$;

$X_{1 e}\left(X^{\prime}{ }_{1 e}\right), X_{2 e}\left(X^{\prime}{ }_{2 e}\right), \ldots, X_{n e}\left(X_{n e}^{\prime}\right)$ - input signals for switching from state $A_{1}\left(A_{1}^{\prime}\right), A_{2}\left(A_{2}^{\prime}\right), \ldots, A_{n}\left(A_{n}^{\prime}\right)$ to state $A_{e}$, respectively;

$t_{0}$ - output signal "start of observation session";

$t^{1}, t^{2}, \ldots, t^{n}$ - output signal "detection time" $1,2, \ldots$, n-th process, respectively, provided that $t^{1}, t^{2}, \ldots, t^{n} \in\left[t_{0}, t_{1}\right)$;

$t^{l^{\prime}}, t^{2^{\prime}}, \ldots, t^{n^{\prime}}$ - output signal "detection time" $1,2, \ldots, \mathrm{n}$-th process, respectively, provided that $t^{l^{\prime}}, t^{2^{\prime}}, \ldots, t^{n^{\prime}} \in\left[t_{1}, t_{\kappa}\right]$

$t_{\kappa}$ - output signal "end of observation session".

It should be noted that in states $A_{1} \ldots A_{n-1}\left(A_{1}^{\prime} \ldots A_{n-1}^{\prime}\right)$, in addition to the estimation problem, the problem of detecting new processes with a frequency of using quanta of $f_{\min s}$ is solved.

The automaton switches to state $A_{n}\left(A_{n}^{\prime}\right)$ when $I$ reaches the threshold value $I^{\prime}$; in this case, the detection of new processes stops, and the resource reserved for detection ( $S$ - quanta) is used to solve the problem of evaluating a process that is in the "priority" mode.

The use of the considered approach to solving the problem of distribution of time resources of the information system when observing the flows of random processes makes it possible to obtain a gain in the estimation accuracy of up to $25 \%$ in comparison with the uniform plan.

\section{References}

1. Boldyrikhin N V, Khutortsev V V 2006 Automation and Telemechanics 12 43-55

2. Khutortsev V V, Boldyrikhin N V 2005 Automatic Control and Computer Sciences 39(5) 9-19 
3. Khutortsev V V 2013 Automatic Control and Computer Sciences 47(1) 48-56

4. Boldyrikhin N V, Rybalko I P, Sosnovskiy I A, Girin I S 2017 Proceedings of the North Caucasus branch of the Moscow Technical University of Communications and Informatics 1(1) 71-73

5. Boldyrikhin N V, Rybalko I P, Sosnovsky I A Yarovoy A V 2013 Proceedings of the North Caucasian branch of the Moscow Technical University of Communications and Informatics 161-65

6. Boldyrikhin N V, Safaryan O A, Razumov P V, Porksheyan V M, Smirnov I A, Korochentsev D A, Cherckesova L V, Romanov A M 2020 ICCAIS 2020 - 3rd International Conference on Computer Applications and Information

7. Boldirikhin N, Safaryan O, Svizhenko A 2019 Radiation and Scattering of Electromagnetic Waves (RSEMW) DOI: 101109/RSEMW20198792788

8. Mealy G H A Bell 1955 System Technical Journal American Telephone and Telegraph Company 34 1045-1079

9. A. O. Lukin and V. Y. Alpatov, Materials Science Forum, Vol. 931, pp. 247-251, 2018. DOI: https://doi.org/10.4028/www.scientific.net/MSF.931.247

10. V. Zubkov, A. Lukin, V. Alpatov MATEC Web Conf. 19601005 (2018). DOI: 10.1051/matecconf/201819601005

11. D. Chernyshev, V. Alpatov, MATEC Web Conf. 11700028 (2017). DOI: 10.1051/matecconf/201711700028 